\title{
ENHANCING WATER AND OIL REPELLENCY OF TEFLON SURFACE BY IMPARTING MICRO-ROUGH STRUCTURES USING SIMPLE ONE-STEP
}

\author{
Khedir R. Khedir \\ Dept. of Physics, College of Science, University of Duhok, Kurdistan Region, Iraq - (khedir.ramazan@uod.ac)
}

\begin{abstract}
:
In this work, an easy one-step and inexpensive technique of mechanical wet sanding was used to impart micro structures into the Teflon surface that promotes super repellent properties toward water and the two moderate low surface tension organic liquids. Sandpapers with a wide range of grit sizes 60-1000, with associated particle sizes of 256-10 $\mu \mathrm{m}$, were used to obtain physical modification of the Teflon surface. The roughened Teflon surface with the sandpaper of 400 grit size showed super repellency toward water, glycerol, and ethylene glycol with CAs as high as $158^{\circ}, 150^{\circ}$, and $142^{\circ}$, respectively, as well as low sliding angle of less than $2^{\circ}, 5^{\circ}$, and $15^{\circ}$, respectively. The obtained results and the effect of roughness were explained in terms of both fundamental wetting models of Wenzel and Cassie-Baxter. The effect of decrease in liquid surface tension on the length scale of imparted geometries and consequent wetting state was also concluded. Finally, the work of adhesion for the tested liquids while on the roughened Teflon surfaces were also determined using both Young-Dupre relation and the liquid's SAs.
\end{abstract}

KEYWORDS: Teflon, Mechanical sanding, Roughness, Wettability, Work of Adhesion.

\section{INTRODUCTION}

The past decade has witnessed numerous studies in the fabrication of water super-repellent surfaces, super hydrophobic surfaces with $\mathrm{CA}>150^{\circ}$, using various techniques and materials (Bhushan and Nosonovsky, 2007; Gao and Macarthy, 2006; Lafuma and Quere, 2003; Nilsson et al, 2010; Patankar 2009). Several studies were conducted for the fabrication of surfaces that could be super-repellent towards water and a diverse range of low surface tension liquids such as organic liquids, superomniphobic surfaces, but are very limited (Cao et al, 2008; Chien-Te et al, 2009; Deng et al, 2012; Tuteja et al, 2007). Decrease in surface tension of liquids compared to the high surface tension of water has made the development of such surfaces more challenging. Nevertheless, developing such surfaces would significantly expand the range of applications of superrepellent surfaces which include self-cleaning and dirt free purposes of smart screens, solar panels, anti-fouling, dragfree, anti-icing, biotechnology instruments, and military devices.

The interaction of liquids with smooth solid surfaces in terms of interfacial energies is described by Young model $\left(\cos \theta_{Y}=\gamma_{s v}-\gamma_{s l} / \gamma_{l v}\right)$, where $\theta_{Y}$ is intrinsic contact angle (CA) of liquids over the smooth surface and $\gamma$ represents the interfacial energies $(s, v$, and $l$ stands for solid, vapor, and liquid phases, respectively). Therefore, the liquid's CA on the surface is the measure of the surface wetting properties. A solid surface that maintains liquid's CA less than $90^{\circ}$ is the indication of the surface high wettability, and poor wettability is when the CA is more than $90^{\circ}$. Superrepellency property of solid surfaces toward a specific liquid is obtained when the liquid maintains a CA of more than 150 degrees.

The maximum CA observed on a smooth solid surface is for water $\left(\gamma_{l v}=72.1 \mathrm{mNm}^{-1}\right)$ while in contact with Teflon surface which is around $120^{\circ}$. The high CA is due to the Teflon's low surface energy $\left(\gamma_{s v}<20 \mathrm{mNm}^{-1}\right)$. However, for lower surface tension liquids such as Glycerol $\left(\gamma_{l v}=63 \mathrm{mNm}^{-1}\right)$ and ethylene glycol $\left(\gamma_{l v}=47.3 \mathrm{mNm}^{-1}\right)$, the CA on the Teflon surface is dramatically decreases to be only around $90^{\circ}$ for both liquids. Thus, even Teflon surface gets wet while in contact with low surface tension organic liquids. This fact can be noticed in Young equation as well, where the CA of liquids on a specific surface is the function of liquid's surface tension $\left(\theta_{Y} \alpha \cos ^{-1}\left(1 / \gamma_{l v}\right)\right)$, in addition to the substrate's surface energy $\left(\gamma_{s v}\right)$.

Roughness and low surface energy of solid surfaces is the key to amplify their repellency toward liquids. The effect of roughness on the wetting properties of surfaces is described by Wenzel model $\left(\cos \theta=r \cos \theta_{Y}\right)$, where $r$ is the ratio of surface roughness and is always greater than one, $\theta$ is the liquid's CA over the rough surface. Super repellency properties can only be obtained by developing a surface roughness that allows air packets to be trapped in its cavities, promoting the liquid to sit on the top of rough features without invading the cavities. This scenario of liquid-surface interaction is described by Cassie model $\left(\cos \theta=\varphi\left(1+\cos \theta_{Y}\right)-1\right)$, where $\varphi$ is the ratio of contact area between liquid and solid surface, while $\theta$ is the liquid's CA when the Cassie model is adopted. Low sliding angle (SA) of less than 10 degrees for liquid droplets while on the tilted surface is another property of Cassie state.

Teflon as a fluoropolymer is well-known for its high resistance to chemicals, heat, weather conditions, and moisture absorption as well as its toughness and flexibility. Given all these exceptional properties, Teflon is widely used for numerous applications. However, Teflon surface with super repellency properties toward various liquids would significantly widen its range of applications. For this purpose, there has been very limited number of studies which mainly focused on imparting super repellency only toward water. Dry mechanical sanding of Teflon surface and its water repellency properties were first conducted by Nilsson et al. (Nilsson 2010). In their study, micro 
scale roughness was imparted to Teflon surface using grit designation of 40-600, obtaining water $\mathrm{CA}$ as high as $150^{\circ}$ and $\mathrm{CAH}$ as low as $4^{\circ}$. In another study by the same group, considering superhydrophobic properties of sanded Teflon, various grit sizes are used to change the $\mathrm{CAH}$ of the Teflon surface while retaining the same advancing CA (Nilsson 2012). It was shown that the variation in Teflon CAH caused deflection in the water droplet trajectory as well as its speed while moving over the surface. Farshchian and co-workers (Farshchian, 2010) used both sanding the poly (methyl methacrylate) surface with a sandpaper of 240 grit size for developing micro scale structures and post treatment with $\mathrm{O}^{2}$ plasma to develop nano scale structures over micro scale roughness obtained from sanding. After salinization of the surfaces, water CA as high as $165^{\circ}$ was obtained. This simple technique of sanding has also been used to produce drag free surfaces of Teflon as a result of the surfaces of the superhydrophobic property (Song et al, 2014). Using a different approach, Liam et al. (Liam, 2016) deposited Teflon onto surfaces using spin coating technique; high superhydrophobic surfaces with water CA of more than $170^{\circ}$ were obtained.

To the best of our knowledge, no studies have been conducted so far for developing a Teflon surface with super repellency toward low surface tension organic liquids, particularly using easy and inexpensive techniques such as mechanical sanding. In the present work, in addition to superhydrophobicity, highly oleophobic Teflon surface toward moderately low surface tension liquids such as Glycerol (GC) and Ethylene Glycol (EG) was developed by an easy and one-step wet mechanical sanding. Unlike dry sanding, mechanical wet sanding contributed in developing super repellent Teflon surfaces using larger grit sizes of 400800 , smaller particle sizes. In addition to the robustness of such surfaces from wearing and preventing small size droplets to invade the gaps, the surfaces still look smooth. Teflon surface which is super repellent toward water as well as relatively low surface tension liquids such as GC and EG would significantly widen the range of its applications.

\section{MATERIALS AND METHODS}

For this study, commercially available Teflon (PTFE) sheets (2" diameter) manufactured by DuPont and sandpapers (3M abrasives) with grit sizes ranging from 60 to 1000 were used. The Teflon sheets were mounted on a horizontal surface and then mechanical wet sanding was carried out in the presence of acetone on the surface. The wet sanding using particular grit designation was conducted applying normal force with no preferred horizontal direction for time duration of 20-30 seconds. Excessive force or sanding for a long period of time would remove the features already imparted. To obtain consistent results, 5 samples were prepared per each grit designation. Finally, the treated surfaces were again washed with acetone and blown with compressed air.

A field emission scanning electron microscope JSM-7000F (JEOL USA, Inc) was used to analyze the morphological properties of the untreated and modified Teflon surfaces. Due to the insulating properties of Teflon, all the surfaces were coated with a thin layer of gold to ground the sample. The SEM images were obtained at working distance of 6 $10 \mathrm{~mm}$ and an accelerating voltage of $15 \mathrm{kV}$.

Wetting analysis of the generated surfaces using various liquids with different surface tensions was carried out using VCA-Optima goniometer. For measuring the CA of the dispensed liquids on the samples, the obtained CAs from three measurements per each sample were averaged. For the accuracy of data, the CAs obtained from more than 5 samples were taken under consideration. The SAs were measured by tilting the samples until gently dispensed droplet of $20 \mu \mathrm{l}$ begins to slide. For each liquid, the SAs were measured after the samples were cleaned with acetone and then dried off with compressed air. The observed SAs of three times of three different locations for each liquid were averaged and reported.

\section{RESULTS AND DISCUSSION}

Table 1 shows the measured values of the three liquids' contact angles and their sliding angles on the untreated Teflon surfaces. The surface observed a CA of around $120^{\circ}$ for water, which is the highest among all other smooth material surfaces. Meanwhile, the Teflon surface observed CAs around $90^{\circ}$ for both GC and EG with surface tensions of 63 and $47.3 \mathrm{mN} / \mathrm{m}$, respectively. This shows that even Teflon is poor in repelling the lower surface tension liquids compared to water with the high surface tension of $72.8 \mathrm{mN} / \mathrm{m}$. The decrease in CA of the liquids with the decrease in their surface tension on the same smooth solid surface is consistent with Young's model.

The associated average particle diameter on the sand papers to the sandpaper grit sizes (GS) is shown in Figure 1. The average size of the particle's diameter decreases exponentially with the increase of GS sandpaper. More than $250 \mu \mathrm{m}$ particle diameter associates to the smallest GS of 60 and the $10 \mu \mathrm{m}$ particle diameter is for the highest GS of 1000 .

Table (1): The three liquids (DIW, GC, and EG) surface tensions, CAs, and SAs for untreated Teflon sheet.

\begin{tabular}{llll}
\hline \multicolumn{1}{c}{ Liquid } & $\begin{array}{c}\text { Surface } \\
\text { Tension } \\
(\mathrm{mN} / \mathrm{m})\end{array}$ & $\begin{array}{c}\text { Liquid CA } \\
\left({ }^{\circ}\right)\end{array}$ & Liquid SA $\left(^{\circ}\right)$ \\
\hline Water & 72.1 & $112 \pm 3$ & $35 \pm 2$ \\
\hline Glycerine & 63 & $95 \pm 4$ & $33 \pm 3$ \\
\hline $\begin{array}{l}\text { Ethylene } \\
\text { Glycol }\end{array}$ & 47.3 & $98 \pm 4$ & $30 \pm 3$ \\
\hline
\end{tabular}

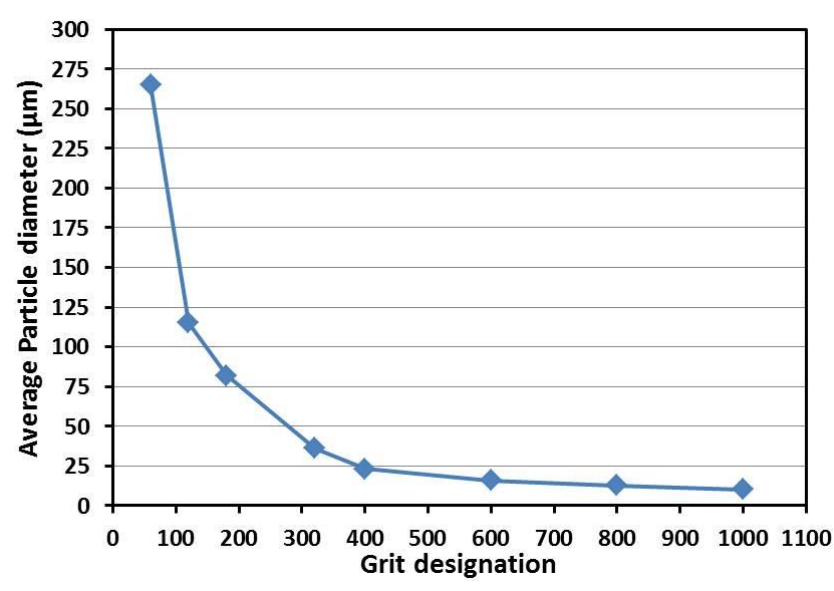

Figure 1. Average particle diameter of the sandpapers associated to their GS (Nilsson 2010)

Figure 2 demonstrates typical SEM images of the untreated smooth Teflon surfaces as well as wet sanded Teflon surfaces using three different GSs of 60, 400, and 1000. A significant variation can be noticed in the morphology of the surfaces considering the size of the micro structures and the gaps in between the structures. Untreated Teflon surface looks quite smooth; however, its surface does have visible regular lines which apart by millimeters. These scratch-like lines could be the result of manufacturing while being cut and have no significant effect on the surface wetting properties. This is due to the fact that the lines are apart by dimensions comparable to the diameter of the droplet. Also the DIW CA of less than $120^{\circ}$ is another 
evidence of no effect on the surface wetting properties due to those scratch-like lines.

Wet mechanical sanding of the Teflon surface with 60 GS sandpaper resulted in imparting large size geometries with dimensions more than $50 \mu \mathrm{m}$ and spaces of more than $70 \mu \mathrm{m}$. The developed large geometries on the Teflon surface are consistent with the large size particles of the associated sandpaper of $60 \mathrm{GS}$. The dimensions of the geometries and spaces in between are reduced with the increase in sandpaper GSs, where for 1000 GS the geometries on the Teflon surface are less than $10 \mu \mathrm{m}$ in size, in addition to the gaps of significantly less than $10 \mu \mathrm{m}$ size. For the moderate case of $400 \mathrm{GS}$, geometries and gaps with dimensions of around 20$30 \mu \mathrm{m}$ were obtained. This is due to the fact that sandpaper with larger GS has smaller particle size which consequently imparts structures with smaller dimensions into the Teflon surface.
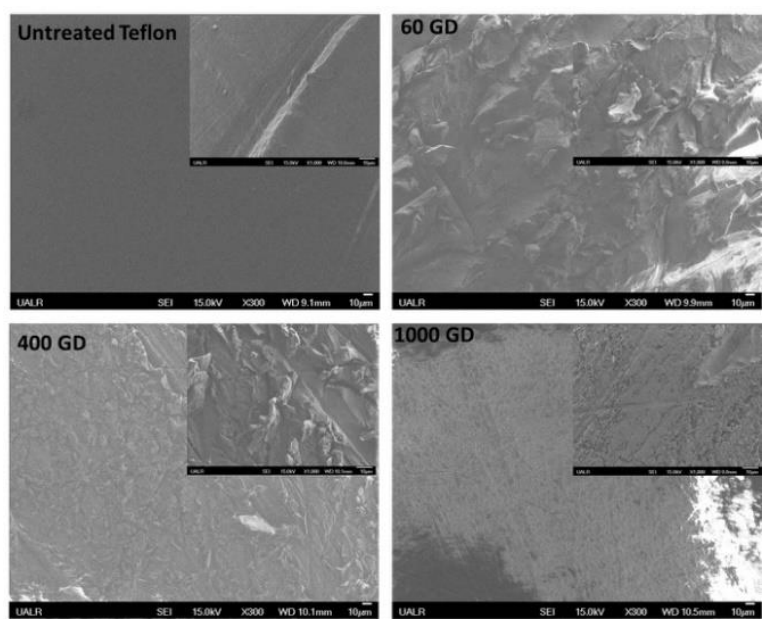

Figure 2. SEM images of the untreated Teflon surface and a three typical wet mechanical sanded Teflon surfaces using 60, 400, and 1000 grit sizes with X300 magnification. Insets are the higher magnification (X1000) of the associated SEM images.

The effect of easy one-step approach of wet mechanical sanding on the Teflon surface wetting properties of the three tested liquids (DIW, GC, and EG) is shown in Figure 3a. It shows the CAs of the three liquids on the physically modified Teflon surface by wet mechanical sanding using a wide range of sandpaper GS. It can be noticed that the surface roughness of the Teflon surface has enhanced its repellency toward the three tested liquids (Nilsson 2010). This is in accordance with Wenzel model which implies that the increase in roughness amplifies the wetting properties of solid surfaces. For the three liquids the $\theta_{\mathrm{Y}}$ of the untreated smooth Teflon surface, as listed in table (1), is more than $90^{\circ}$ for DIW and GC, and around $90^{\circ}$ for EG. Therefore, the increase in CAs of the modified Teflon surfaces is consistent with Wenzel model. However, the transition from sticky Wenzel state, due to invasion of liquids into the gaps, to the slippery Cassie state, liquids bridging over the top of structures, depends on the size of structures and the size of spaces between the structures (aspect ratio).

For the three liquids, CAs started to increase dramatically with the increase in sandpaper GS reaching 400, but after 400 GS, the CAs started to decrease less dramatically. In terms of their liquid surface tensions, lower surface tension liquids started with lower CAs. Typical digital images of the liquid droplets covering the whole range are demonstrated in Figure 4. The lowest CAs are obtained for the two extremes of 60 and $1000 \mathrm{GS}$, while the optimal super-repellency toward the three liquids was only obtained when 400 GS sandpaper was used to roughen the Teflon surface. For the two high surface tension liquids of DIW and GC, excluding the two extremes of GS, most of the modified Teflon surface showed super repellency. However, in the case of EG, relative super repellency of Teflon surface was obtained only when $400 \mathrm{GS}$ sandpaper was used.
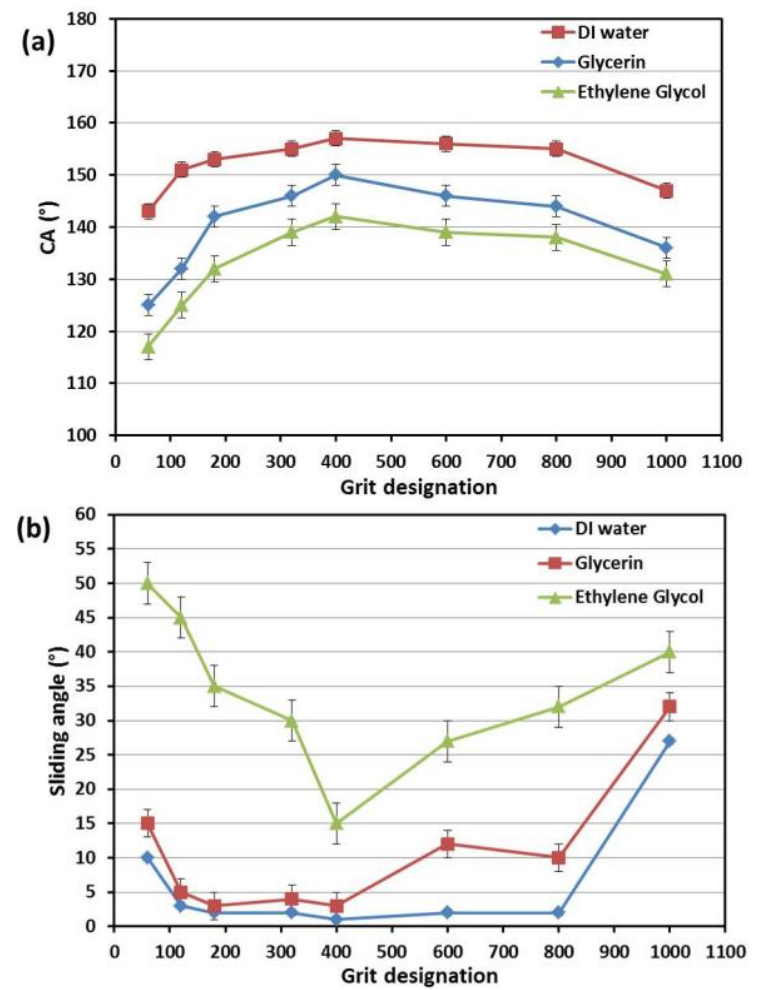

Figure 3. Observed CAs of the three different liquids of DIW, GC, and EG on the modified Teflon surfaces versus the utilized various sandpaper GS to roughen their surfaces (a). Also the Teflon surface sliding angles of the three liquid droplets versus various sandpaper GS used to roughen its surface (b).

The dynamic analyses for the three tested liquids (DIW, GC, and EG) over the modified Teflon surfaces using the wide range of sandpaper GSs was carried in terms of SA measurements of the liquid droplets. The observed SAs for the three liquids as the function of sandpaper GS used to roughen Teflon surfaces are demonstrated in Figure (3b). The liquid SAs on the physically modified Teflon surfaces are highest at the two extremes; while relatively lower in between and lowest at 400 GS. For both DIW and GC, the SAs are significantly lower compared to EG SAs, while DIW SAs are consistently low, less than 10 degrees, for most of the sandpaper GS but at 1000 GS. In the case of EG, the SAs are significantly high and even higher than its SA on the smooth Teflon surface. Lowest SA of around 15 degrees for EG was only obtained at $400 \mathrm{GS}$.
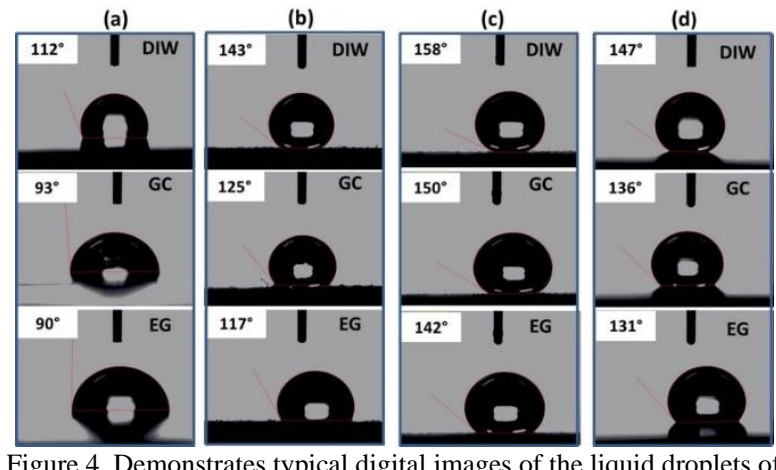

Figure 4. Demonstrates typical digital images of the liquid droplets of $10 \mu \mathrm{L}$ DIW, GC, and EG captured on the surface of untreated smooth Teflon (column (a)) and roughened Teflon surfaces using wet 
mechanical sanding of grit sizes of 60 (column (b)), 800 (column (c)), and 1000 (column (d)).

Form the analyses of both static (CAs) and dynamic (SAs) wetting properties of the modified Teflon surfaces (Figure (3), the aspect ratios of the imparted structures by the various particle sizes $(260-10 \mu \mathrm{m})$ of different sandpaper GSs $(60$ 1000 ), are responsible for adopting a particular wetting state by the surfaces. In the case of untreated Teflon surface, Table (1), the SAs for the three liquids were more than 30 degrees, however relatively less for lower surface tension liquids in spite of smaller $\theta_{Y}$.Lower SAs for the two organic liquids compared to DIW which exhibited higher CA can be described in terms of work of adhesion $\left(W_{a}\right)$. For the liquid droplet to slide over the solid surface, it has to overcome the $W_{a}$ that arises at the contact area as a result of contact angle hysteresis $(\mathrm{CAH})$. As the outcome of both physical and chemical heterogeneity of the surface, $\mathrm{CAH}$ is the measure of the stickiness of liquid droplet to the surface. According to Young-Dupre relation, the $W_{a}$ which is the function of both $\gamma_{l v}$ and $\theta$ can be expressed as:

$$
W_{a}=\gamma_{l v}\left(1+\cos \theta_{Y}\right)
$$

Considering the above relation (eq. (2)), $W_{a}$ for the three liquids is: $45.5,57.5,48.1 \mathrm{mN} / \mathrm{m}$ for DIW, GC, and EG, respectively. However, in terms of $W_{a}$ DIW must show lower SA compared to the two other organic liquids, but this could be due to the high polarity of DIW that promotes stronger adhesion to the surfaces in spite of smaller contact area at the interface. The lower SA for EG compared to GC in spite of its higher CA, less contact area is consistent to the calculated $W_{a}$ for both liquids.

Wet mechanical sanding with small GSs or larger particle sizes that led to higher degree of roughness is promoting partial Cassie state for both high surface tension liquids of DIW and GC. For significantly lower surface tension liquid of EG, large SA of 30-50 degrees is due to the invasion of gaps by the liquid promoting the wetting state of Wenzel. The larger SAs of EG compared to its SA on the corresponding smooth surface is the sign of larger area of contact with the liquid. The effect of surface tension in promoting a particular state of wetting can be described in terms of the balance in Laplace pressure difference at the interface that bridges over the gaps. The Young-Laplace relation describes the pressure balance at the interface in terms of liquid surface tension and the interface curvature $(R)$ as follows (Xiu, 2008; Chhatre, 2010):

$$
\Delta P=2 \gamma_{l v} / R
$$

However, this is only a hypothesis because Laplace pressure can only be considered when the capillary action occurs, which depends on the capillary length $\left(\lambda_{c}=\gamma_{l v} / \rho g\right)^{1 / 2}$ for each liquid. The $\lambda_{c}$ values are 2.7 and $2.0 \mathrm{~mm}$ for DIW and EG, respectively. These values of $\lambda_{c}$, even for EG, are still significantly larger than the length scale of the imparted geometries even for the case of $60 \mathrm{GS}$ sandpaper. Therefore, the effect of capillary action and unbalance in Laplace interfacial pressure that could drive the transition from dried state of Cassie to wetting state of Wenzel would remain only a bare hypothesis. More practical explanation can be made by introducing the optimal roughness $\left(r=\frac{\varphi-1}{\cos \theta_{c}}+\varphi\right)$ and length scale that promotes Cassie state. In this case the adoption of Wenzel state is due to an extreme liquid meniscus at the interface as a result of wide spacing of the geometries, and eventually touching the bottom of the cavities with lower surface energy minima preferring invasion of the gaps (Patankar, 2004).

The significant decrease in the EG surface tension could develop such extreme concavity that causes unbalanced pressure at the interface leading to the invasion of gaps by the liquid. Chhatre and co-workers (Chhatre et al, 2010) also concluded that geometries with small distances in between are more robust in adopting Cassie state for low surface tension liquids. Due to their significantly higher surface tensions, both DIW and GCcan bridge over the gaps without being pulled down. In the case of wet sanding of Teflon surfaces using large GSs, lower degree of roughness, the decrease in liquids CAs and increase in the SAs is due to the fact that the aspect ratios of the structures are not large enough to trap enough air to maintain the balance in Laplace pressure that could prevent the liquids from invading the gaps.

For the surfaces that are in Cassie state or partial wetting (combination of Cassie and Wenzel state), the $W_{a}$ can be calculated by considering the solid fraction $\left(\varphi=\frac{1+\cos \theta}{1+\cos \theta_{Y}}\right)$ in contact with the liquid as following (Hsieh 2009):

$$
W_{a}=\varphi \gamma_{l v}\left(1+\cos \theta_{Y}\right)
$$

in a simpler way, by substituting the relation for $\varphi$ in equation (3), $W_{a}$ for a rough surface can be represented as:

$$
W_{a}=\gamma_{l v}(1+\cos \theta)
$$

For both liquids DIW and GC, the droplets are mostly in the Cassie state (middle section of the CAs curve) or partial Cassie state (at extremes of CAs curve). Also a smaller SA of the liquids compared to their SAs while on the smooth surface is another indication of adopting Cassie or partial wetting of Cassie-Wenzel state. If the SA of a droplet on a rough surface is larger than its SA while on the corresponding smooth surface, it indicates that the surface has adopted the Wenzel state of complete wetting that promotes larger contact area and consequently higher adhesion. The wetting analyses of solid surfaces cannot be comprehensive without considering their dynamic wetting properties. Considering the liquid's SAs, they would give us more realistic calculations in terms of work of adhesion at which the droplet needs to overcome and begin sliding over the surface. Two different surfaces with similar CAs can promote different dynamic behavior of droplets depending on their $\mathrm{CAH}$; the difference between advancing CA $\left(\theta_{a}\right)$ and receding CA $\left(\theta_{r}\right)$. Lotus effect (Nosonovsky et al, 2009; Cheng et al, 2006) is the best example of surfaces with high CA but small CAH, small SA, while Petal effect (Feng et al, 2008; Bhushan et al, 2010) is the best example of high CA and large $\mathrm{CAH}$ as well, and consequent large SA. For the liquid droplet to slide by the effect of its weight $(\rho V g)$ after tilting the substrate (SA) by a specific angle of $(\alpha)$, $W_{a}$ can be expressed as (Xiu et al, 2008; Hsieh et al, 2009):

$$
W_{a}=\frac{\rho V g \sin \alpha}{w}
$$

Where $(w=\pi r)$ represents the width of the contact area of liquid with radius $(r)$ over the surface

Figure (5): Calculated work of adhesion as the function of both observed CAs (a) and SAs (b) versus the used sandpaper GS. 
(a)

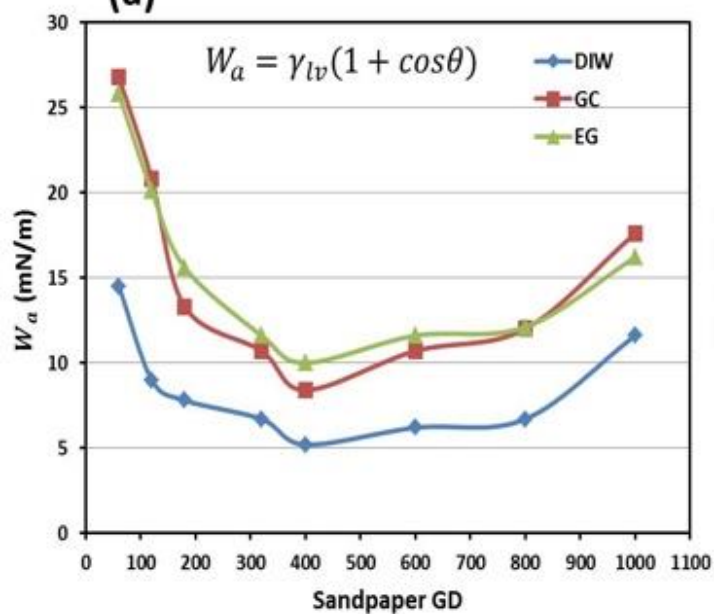

(b)

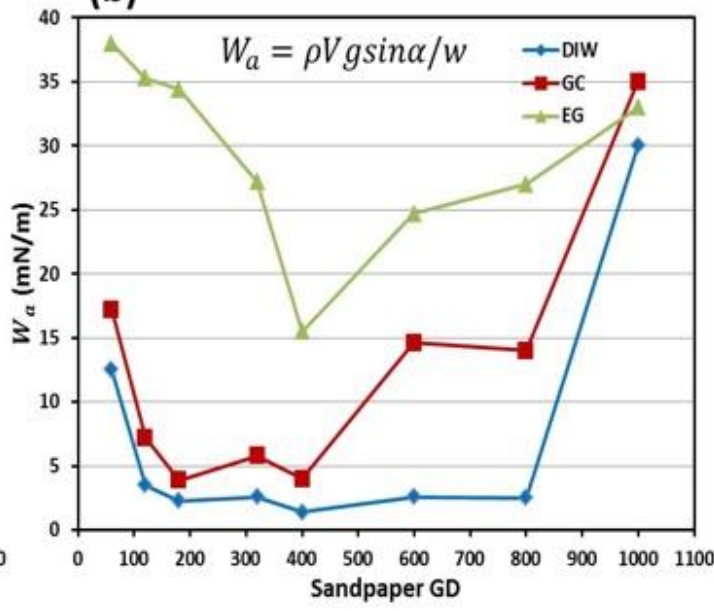

Figure 5. Calculated work of adhesion as the function of both observed CAs (a) and SAs (b) versus the used sandpaper GS

Figure 5 shows the calculated $\mathrm{W}_{\mathrm{a}}$ for the three tested liquids in terms of both Young-Dupre relation and their SAs on the surface of roughened Teflon surface using various sandpaper GSs. Young-Dupre relation is the function of both liquid's surface tensions and their observed CAs without considering the surface $\mathrm{CAH}$, while equation (5) is the function of surface CAH that can also be represented in terms of SAs, such as (Xiu et al, 2008; Hsieh et al, 2009):

$$
\frac{\rho V g \sin \alpha}{w}=\gamma_{l v}\left(\cos \theta_{r}-\cos \theta_{a}\right)
$$

The determined trend of the $W_{a}$ using both equations (4) and (5) and plotted in Figures $5 \mathrm{a}$ and $5 \mathrm{~b}$ is more realistic when CAH embedded in the SAs are considered. In Figure 5a, $W_{a}$ for both GC and EG are overlapped in spite of significant difference in their SAs as well as CAs. Larger SAs is the manifestation of higher adhesion to the surface that the liquid droplet needs to overcome and begin sliding. Figure $5 \mathrm{~b}$ also shows the significance increase in $W_{a}$ for $\mathrm{EG}$ on the roughened Teflon surfaces and particularly for large geometries imparted by small GS sandpapers due to wetting Wenzel state. In addition, the values of $W_{a}$ for DIW are significantly low, in the range of few $\mathrm{mN} / \mathrm{m}$, which explains why the DIW droplets were mostly floating on the rough surfaces promoting negligible SAs for the whole range of sandpaper GSs excluding the two extremes.

The highest CAs of $158^{\circ}, 150^{\circ}$, and $142^{\circ}$ and lowest SAs of $1^{\circ}, 3^{\circ}$, and $15^{\circ}$ for DIW, GC, and EG, respectively were obtained on the surface of a modified Teflon surface using $400 \mathrm{GS}$. This is due to the fact that the aspect ratio of the imparted structures promotes a balance in Laplace pressure at the liquid/air interface over the gaps in spite of significant decrease in the EG surface tension. A photograph of the three tested liquid droplets used in this study while dispensed on both untreated Teflon surface and the wet mechanically sanded Teflon surface using $400 \mathrm{GS}$ is demonstrated in Figure 6. A notable difference is visible in the behavior of the two Teflon surfaces in terms of interaction with the three liquids, where the three droplets have taken a spherical shape while on the modified Teflon surface compared to hemispherical shape while on the untreated Teflon surface.

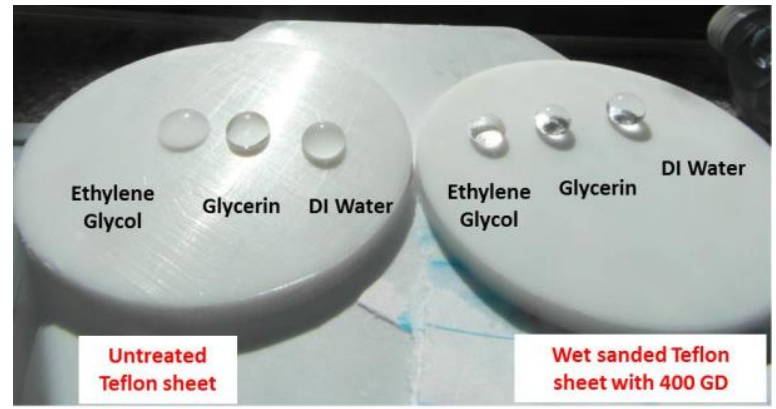

Figure 6. A photograph of $20 \mu \mathrm{L}$ liquid droplets of DIW, GC, and EG dispensed on both untreated Teflon surface and wet sanded Teflon surface using $400 \mathrm{GS}$.

Comparing the results obtained by mechanical wet sanding for water repellency of Teflon surface to the work carried out by Nilsson and co-workers using dry sanding, this study has shown enhancement in the water repellency of Teflon surfaces by exhibiting a CA in the vicinity of 160 degrees for DIW compared to 150 degrees in the case of dry sanding. This could be due to the fact that acetone presence could have removed more residues of sanding, contributing into higher aspect ratios, in addition to the removal of hydrophilic particles of the used sandpapers during the sanding process. In this study the highest CA of $158^{\circ}$ with lowest $\mathrm{SA}$ of $<2^{\circ}$ for water was obtained with the GS of 400 (sandpaper particle size of $\sim 25 \mu \mathrm{m}$ ), which shows improved results compared to the other previous studies (Nilsson et al, 2010) that used no acetone while performing the sanding process. Therefore, in terms of cosmetic view, our super repellent Teflon surface still looks smooth and even nicer than the untreated one, as demonstrated in Figure 6. In addition, this study has covered the oil-repellency of Teflon surfaces as well as the effect of liquid's surface tension. It was shown that the moderately fine sandpaper with 400 GS can make a significance enhance in the repellency of Teflon surfaces toward moderate low surface tension liquids. For extreme low surface tension liquids, more sophisticated techniques in terms of imparting re-entrant structures are underway.

\section{CONCLUSIONS}

In this work, an easy one-step and inexpensive technique of wet mechanical sanding is utilized using wide range of sandpaper GSs (60-1000) of Teflon attributed to super repellency of Teflon surface toward relatively low surface tension organic liquids such as ethylene glycol. Physically modified Teflon surface using 400 GS sandpaper showed super repellency toward the three tested 
liquids. This simple technique of wet sanding can be used to develop rough surfaces with a wide range of particle geometries without using complicated techniques for the study of interaction of those surfaces with different surface tension liquids. Teflon surface with super repellency properties toward water and organic liquids in addition to its chemical resistivity and physical robustness would be more functional and compatible to the surrounding environment.

\section{ACKNOWLEDGEMENTS}

The author would like to thank Dr. Tansel Karabacak and his group from Department of Physics and Astronomy at UALR, USA, for conducting CA measurements. Also help from the Center for Integrative Nanotechnology Sciences at UALR, USA, for capturing the SEM images is highly appreciated.

\section{REFERENCES}

Bhushan, B., and Nosonovsky, M. (2007). Hierarchical Roughness Makes Superhydrophobic States Stable. Microelectronic Engineering, 84, 382-6.

Bhushan, B., and Nosonovsky, M. (2010). The Rose Petal Effect and the Modes of Superhydrophobicity Philosophical Transactions of the Royal Society London, Series A: Mathematical, Physical and Engineering Sciences, 368, 4713-28.

Bhushan, B.; Jung, Y. C.; Koch, K. Micro-, Nano- and Hierarchical Structures for Superhydrophobicity, Self-Cleaning and Low Adhesion. Philosophical Transactions of the Royal Society A: Mathematical, Physical and Engineering Sciences 2009, 367, 1631-1672.

Cao, L.; Price, T. P., Weiss, M., Gao, D. (2008). Super Water- and Oil-Repellent Surfaces on Intrinsically Hydrophilic and Oleophilic Porous Silicon Films. Langmuir, 24, 16401643.

Cheng, Y. T., Rodak, D. E., Wong, C. A., Hayden, C. A. (2006). Effects of Micro- and Nano-Structures on the SelfCleaning Behaviour of Lotus Leaves. Nanotechnology, 17, 1359-1362.

Chhatre, S. S., Choi, W., Tuteja, A., Park, K., Mabry, J. M., McKinley, G. H., Cohen, R. E. (2010). Scale Dependence of Omniphobic Mesh Surfaces. Langmuir, 26, 40274035 .

Chien-Te, H., Fang-Lin, W., Wei-Yu C. (2009). Super Water- and Oil-Repellencies from Silica-Based Nanocoatings. Surf. Coat. Technol., 203, 3377-84.

Deng, X., Mammen, L., Butt, H., Vollmer, D. (2012). Candle Soot as a Template for a Transparent Robust Superamphiphobic Coating. Science, 335, 67-70.

Farshchian, B., Ok, J. T., Hurst, S. M., Park, S. (2010). Simple fabrication of hierarchical structures on a polymer surface; In NSTI Nanotechnology Conference and Expo, Anaheim, CA, (pp 665-668), USA

Feng, L., Zhang, Y., Xi, J., Zhu, Y., Wang, N., Xia, F., Jiang, L. (2008). Petal Effect: A Superhydrophobic State with High Adhesive Force. Langmuir, 24, 4114-4119.

Gao, L., and McCarthy, T. J. (2006). A Perfectly Hydrophobic Surface (A/R = 180/180). J. Am. Chem. Soc., 128, 90529053.

Hsieh, C., Wu, F., Chen, W. (2009). Contact Angle Hysteresis and Work of Adhesion of Oil Droplets on Nanosphere Stacking Layers. J. Phys. Chem. C, 113, 13683-13688.

Lafuma, A., and Quere, D. (2003). Superhydrophobic States. Nature Materials, 2, 457-60.

Liam R. J. Scarratt, Ben S. Hoatson, Elliot S. Wood, Brian S. Hawkett, and Chiara N. (2016). Durable Superhydrophobic Surfaces via Spontaneous Wrinkling of Teflon AFACS Appl. Mater. Interfaces, 8, 6743-6750.

Nilsson, M. A., and Rothstein, J. P. (2012). Using Sharp Transitions in Contact Angle Hysteresis to Move, Deflect, and Sort Droplets on a Superhydrophobic Surface. Phys. Fluids, 24, 062001.

Nilsson, M. A., Daniello, R. J., Rothstein, J. P. (2010). A Novel and Inexpensive Technique for Creating Superhydrophobic
Surfaces using Teflon and Sandpaper. J. Phys. D, 43, 045301.

Nosonovsky, M., and Bormashenko, E. (2009). In Lotus Effect: Superhydrophobicity and Self-Cleaning; Functional Properties of Bio-Inspired Surfaces Characterization and Technological Applications; World Scientific Publishing Co. Pte. Ltd, Singapore, pp 43-78.

Patankar, N. A. (2004) Transition between Superhydrophobic States on Rough Surfaces. Langmuir, 20, 7097-7102.

Patankar, N. A. (2009). Hydrophobicity of Surfaces with Cavities: Making Hydrophobic Substrates from Hydrophilic Materials? J. Adhes. Sci. Technol., 23, 413-33.

Song, D., Daniello, R.J. \& Rothstein, J.P. (2014). Drag reduction using superhydrophobic sanded Teflon surfaces. J. P. Exp Fluids, $55,1783$.

Tuteja, A., Choi, W., Ma, M., Mabry, J. M., Mazzella, S. A., Rutledge, G. C., McKinley, G. H., Cohen, R. E. (2007). Designing Superoleophobic Surfaces. Science, 318, 1618-22.

Xiu, Y., Zhu, L., Hess, D. W., Wong, C. P. (2008). Relationship between Work of Adhesion and Contact Angle Hysteresis on Superhydrophobic Surfaces. J. Phys. Chem. C, 112, 11403-7. 